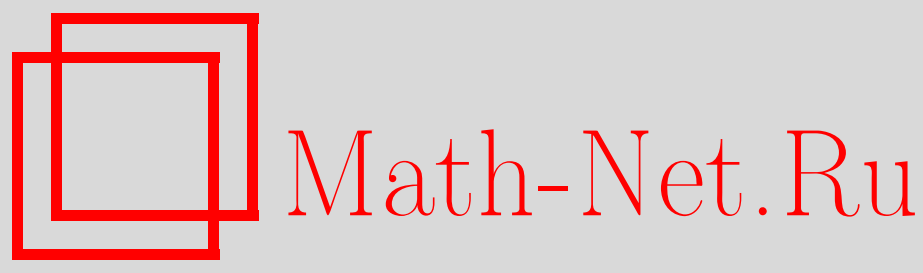

Ю. Х. Эшкабилов, Эффект Ефимова для одного модельного "трехчастичного" дискретного оператора Шредингера, ТМФ, 2010, том 164, номер 1, 78-87

DOI: https://doi.org/10.4213/tmf6525

Использование Общероссийского математического портала Math-Net.Ru подразумевает, что вы прочитали и согласны с пользовательским соглашением http://www . mathnet.ru/rus/agreement

Параметры загрузки:

IP : 3.89 .185 .249

26 апреля 2023 г., 13:39:05

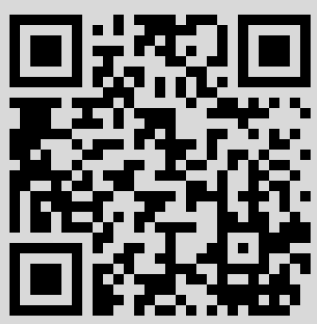




\title{
ФИЗИКА
}

Том 164, № 1

июль, 2010

2010 г.

Ю. Х. Эшкабилов*

\section{ЭФФЕКТ ЕФИМОВА ДЛЯ ОДНОГО МОДЕЛЬНОГО “ТРЕХЧАСТИЧНОГО" ДИСКРЕТНОГО ОПЕРАТОРА ШРЕДИНГЕРА}

\begin{abstract}
Изучается существование бесконечного числа собственных значений одного модельного "трехчастичного" оператора Шредингера $H$. Доказывается теорема о необходимом и достаточном условиях существования бесконечного числа собственных значений в модели $H$ ниже нижнего края существенного спектра.
\end{abstract}

Ключевые слова: существенный спектр, дискретный спектр, эффект Ефимова.

\section{1. ВВЕДЕНИЕ}

Дискретные спектры операторов Шредингера являются наиболее интенсивно изучаемым объектом в теории операторов. Один из важных вопросов в спектральном анализе операторов Шредингера - изучение бесконечности числа собственных значений, лежащих вне существенного спектра, т.е. существование эффекта Ефимова в данной модели. Этот эффект впервые был обнаружен Ефимовым [1] для трехчастичных операторов Шредингера в непрерывном пространстве. Строгое математическое доказательство существования эффекта Ефимова было проведено в работе [2], а затем в работах [3], [4] и др.

Насколько нам известно, впервые в работах Лакаева [5], [6] доказано существование эффекта Ефимова для дискретного оператора Шредингера трехчастичной системы методом, основанным на аналитических свойствах определителей Фредгольма. Затем в работах [7]-[10] изучалось существование эффекта Ефимова для трехчастичных дискретных операторов Шредингера методом Соболева (т.е. методом асимптотики) [11].

В настоящей работе изучено существование эффекта Ефимова для одного модельного дискретного "трехчастичного" оператора Шредингера, возникающего в модели Хаббарда. При исследовании эффекта Ефимова мы будем пользоваться инструментами принципа минимакса для ограниченных самосопряженных операторов и свойствами положительных интегральных операторов. В статье главным образом приведены достаточные и необходимые условия существования эффекта Ефимова ниже нижнего края существенного спектра в данной модели.

*Национальный университет Узбекистана им. М. Улугбека, Ташкент, Узбекистан. E-mail: yusup62@rambler.ru 


\section{2. НЕКОТОРЫЕ ОБОЗНАЧЕНИЯ И НЕОБХОДИМЫЕ СВЕДЕНИЯ}

Пусть $\Omega_{1} \subset \mathbb{R}^{d_{1}}, d_{1} \in \mathbb{N}$, и $\Omega_{2} \subset \mathbb{R}^{d_{2}}, d_{2} \in \mathbb{N},-$ компактные множества. Рассмотрим непрерывные функции $k_{1}(x, s)$ на $\Omega_{1}^{2}, k_{1}(x, s)=\overline{k_{1}(s, x)}$, и $k_{2}(y, t)$ на $\Omega_{2}^{2}$, $k_{2}(y, t)=\overline{k_{2}(t, y)}$. В гильбертовом пространстве $L_{2}\left(\Omega_{1} \times \Omega_{2}\right)$ рассмотрим частично-интегральные операторы $T_{1}$ и $T_{2}$ :

$$
\begin{array}{ll}
T_{1} f(x, y)=\int_{\Omega_{1}} k_{1}(x, s) f(s, y) d \nu_{1}(s), & f \in L_{2}\left(\Omega_{1} \times \Omega_{2}\right), \\
T_{2} f(x, y)=\int_{\Omega_{2}} k_{2}(y, t) f(x, t) d \nu_{2}(t), & f \in L_{2}\left(\Omega_{1} \times \Omega_{2}\right) .
\end{array}
$$

Пусть $k_{0}(s, x)$ - произвольная вещественнозначная непрерывная функция на $\Omega_{1} \times \Omega_{2}$. Обозначим через $H_{0}$ оператор умножения на функцию $k_{0}(x, y)$, т.е.

$$
H_{0} f(x, y)=k_{0}(x, y) f(x, y), \quad f \in L_{2}\left(\Omega_{1} \times \Omega_{2}\right) .
$$

Рассмотрим линейный ограниченный самосопряженный оператор

$$
H=H_{0}-T,
$$

действующий в пространстве $L_{2}\left(\Omega_{1} \times \Omega_{2}\right)$, где

$$
T=T_{1}+T_{2}
$$

Оператор $H$ является общей моделью "трехчастичного" дискретного оператора Шредингера, возникающей в модели Хаббарда на примесной решетке (см. работу [12]).

Существенный спектр оператора $H$ в общем виде изучен в работе [12]. В работе [13] рассмотрены существенный и дискретный спектры операторов в модели (1), когда потенциальная функция $k_{0}(x, y)$ имеет специальный вид: $k_{0}(x, y)=u(x)+h(y)$.

Через $\rho(\cdot), \sigma(\cdot), \sigma_{\mathrm{e}}(\cdot)$ и $\sigma_{\mathrm{d}}(\cdot)$ обозначим соответственно резольвентное множество, спектр, существенный спектр и дискретный спектр самосопряженных операторов [14].

В монографии [15] с помощью принципа минимакса изучены спектральные свойства заданных самосопряженных ограниченных снизу операторов, действующих в сепарабельном гильбертовом пространстве. В частности, доказано существование собственных значений и бесконечность дискретного спектра (т.е. существование эффекта Ефимова) для некоторых многочастичных гамильтонианов. Сначала мы изложим принцип минимакса для ограниченных самосопряженных операторов, которым будем пользоваться при исследовании на конечность или бесконечность дискретного спектра в модели (1).

Пусть $\mathcal{H}$ - сепарабельное гильбертово пространство, $A: \mathcal{H} \rightarrow \mathcal{H}$ - линейный ограниченный самосопряженный оператор. 
Положим

$$
E_{\min }=E_{\min }(A)=\inf \left\{\lambda: \lambda \in \sigma_{e}(A)\right\} .
$$

Имеем $E_{\min }(A) \in \sigma_{\mathrm{e}}(A)$. Число $E_{\min }(A)$ будем называть нижним краем существенного спектра оператора $A$.

Определим вещественное число

$$
S_{\text {min }}=S_{\text {min }}(A)=\inf \{(A x, x): x \in \mathcal{H},\|x\|=1\} .
$$

Тогда $S_{\min }(A) \in \sigma(A)$, и если $S_{\min }(A)<E_{\min }(A)$, то существует элемент $x_{1} \in \mathcal{H}$, $\left\|x_{1}\right\|=1$, такой, что $S_{\min }(A)=\left(A x_{1}, x_{1}\right)$.

Построим ограниченную возрастающую последовательность $\mu_{n}=\mu_{n}(A), n \in \mathbb{N}$, следующим способом:

$$
\begin{aligned}
\mu_{1}(A) & =S_{\min }(A)=\left(A x_{1}, x_{1}\right), \\
\mu_{\kappa}^{(1)}(A) & =\mu_{1}\left(A_{\kappa-1}\right)=S_{\min }\left(A_{\kappa-1}\right), \quad \kappa=2,3, \ldots,
\end{aligned}
$$

где $S_{\min }\left(A_{k}\right)<E_{\min }(A), A_{\kappa}$ - сужение оператора $A$ на подпространство

$$
\mathcal{H}_{\kappa}=\left\{x \in \mathcal{H}:\left(x, x_{i}\right)=0, i=1,2, \ldots, \kappa\right\} .
$$

Здесь $S_{\min }\left(A_{\kappa-1}\right)=\left(A_{\kappa} x_{\kappa}, x_{\kappa}\right), x_{\kappa} \in \mathcal{H}_{\kappa-1},\left\|x_{\kappa}\right\|=1, \kappa=2,3, \ldots$.

Положим

$$
\mu_{n}(A)=\sup \left\{\mu_{1}(A), \mu_{2}^{(1)}(A), \ldots, \mu_{n}^{(1)}(A)\right\}, \quad n \in \mathbb{N} .
$$

Если для некоторого $k_{0} \in \mathbb{N} \cup\{0\}$ имеем $S_{\min }\left(A_{k_{0}}\right)=E_{\min }(A)$, где $A_{0}=A$, то определим $\mu_{k_{0}+m}=E_{\min }(A), m \in \mathbb{N}$, и

$$
\mu_{k}(A)=\sup \left\{\mu_{1}(A), \mu_{2}^{(1)}(A), \ldots, \mu_{k}^{(1)}(A)\right\}, \quad k \leqslant k_{0}, \quad k_{0} \neq 0 .
$$

ТЕОРемА 2.1 (принцип минимакса для ограниченных операторов в операторной форме). Пусть $A: \mathcal{H} \rightarrow \mathcal{H}$ - ограниченный самосопряженный оператор. Тогда для каждого фиксированного $n \in \mathbb{N}$

либо существует $n$ собственных значений (считая вырожденные собственные значения столько раз, какова их кратность), лежащих ниже нижнего края $E_{\min }(A)$ существенного спектра, а $\mu_{n}(A)$ из (3) есть п-е собственное значение оператора $A$ (с учетом кратности),

либо $\mu_{n}(A)$ - нижний край существенного спектра оператора $A$, m.e. $\mu_{n}(A)=$ $E_{\min }(A) ;$ при этом $\mu_{n}(A)=\mu_{n+\kappa}(A)$ для всех $\kappa \in \mathbb{N}$ и существует самое большее $n-1$ собственных значений (с учетом кратности), лежащих ниже нижнего края $E_{\min }(A)$ существенного спектра оператора $A$.

Линейный ограниченный оператор $A: \mathcal{H} \rightarrow \mathcal{H}$ называется положительным и пишется $A \geqslant 0$ или $0 \leqslant A$, если $(A x, x) \geqslant 0$ для всех $x \in \mathcal{H}$.

СлеДСТВИЕ 2.1. Пусть $A, B: \mathcal{H} \rightarrow \mathcal{H}$ - линейные ограниченные самосопряженнысе операторьи, $A \leqslant B(B-A \geqslant 0)$ u $E_{\min }(A)=E_{\min }(B)$. Тогда

$$
\mu_{n}(A) \leqslant \mu_{n}(B), \quad n \in \mathbb{N} \text {. }
$$




\section{3. ВСПОМОГАТЕЛЬНЫЕ УТВЕРЖДЕНИЯ}

Изучим конечность и бесконечность дискретного и существенного спектров самосопряженного оператора $T$ (2). Определим компактные интегральные операторы $K_{1}$ и $K_{2}$, действующие соответственно в $L_{2}\left(\Omega_{1}\right)$ и $L_{2}\left(\Omega_{2}\right)$ по правилам

$$
\begin{aligned}
& \left(K_{1} \varphi\right)(x)=\int_{\Omega_{1}} k_{1}(x, s) \varphi(s) d \nu_{1}(s), \\
& \left(K_{2} \phi\right)(y)=\int_{\Omega_{2}} k_{2}(y, t) \phi(t) d \nu_{2}(t) .
\end{aligned}
$$

Оператор $T$ является унитарно-эквивалентным оператору $K=K_{1} \otimes E+E \otimes K_{2}$, действующему в пространстве $L_{2}\left(\Omega_{1}\right) \otimes L_{2}\left(\Omega_{2}\right)$ (см. работы [12], [16]), где $E$ - тождественный оператор, $\otimes-$ тензорное произведение. Следовательно, получим $\sigma(T)=$ $\sigma\left(K_{1}\right)+\sigma\left(K_{2}\right)[14]$. Отсюда имеем [16]

$$
\begin{aligned}
& \sigma_{\mathrm{e}}(T)=\sigma\left(K_{1}\right) \cup \sigma\left(K_{2}\right)=\{0\} \cup \sigma_{\mathrm{d}}\left(K_{1}\right) \cup \sigma_{\mathrm{d}}\left(K_{2}\right), \\
& \sigma_{\mathrm{d}}(T)=\left\{\omega: \omega=\alpha+\beta \notin \sigma\left(K_{1}\right) \cup \sigma\left(K_{2}\right), \alpha \in \sigma_{\mathrm{d}}\left(K_{1}\right), \beta \in \sigma_{\mathrm{d}}\left(K_{2}\right)\right\} .
\end{aligned}
$$

Легко заметить, что мощность множеств $\sigma_{\mathrm{e}}(T)$ и $\sigma_{\mathrm{d}}(T)$ не более чем счетна. Из представления существенного и дискретного спектров оператора $T$ легко следует

ПРЕДЛОЖЕНИЕ 3.1. Имеют место следующие неравенства:

$$
\begin{aligned}
\left|\sigma_{\mathrm{d}}\left(K_{\kappa}\right)\right|+1 & \leqslant\left|\sigma_{\mathrm{e}}(T)\right|, \quad \kappa=1,2, \\
\left|\sigma_{\mathrm{e}}(T)\right| & \leqslant\left|\sigma_{\mathrm{d}}\left(K_{1}\right)\right|+\left|\sigma_{\mathrm{d}}\left(K_{2}\right)\right|+1, \\
\left|\sigma_{\mathrm{d}}(T)\right| & \leqslant\left|\sigma_{\mathrm{d}}\left(K_{1}\right)\right| \cdot\left|\sigma_{\mathrm{d}}\left(K_{2}\right)\right|,
\end{aligned}
$$

где $|\ldots|$ - мощность множества.

ПреДЛОЖЕНИЕ 3.2. Дискретный спектр (существенный спектр) оператора Т конечен тогда и только тогда, когда дискретный спектр компактных операторов $K_{1}$ и $K_{2}$ конечен.

ДокАзАтельство. Достаточность очевидна. Докажем необходимость. Допустим обратное: дискретный спектр оператора $T$ конечен, а дискретный спектр оператора $K_{1}$ или $K_{2}$ бесконечен. Надо подчеркнуть, что дискретный спектр самосопряженных компактных операторов состоит из всех их собственных значений, отличных от нуля. Не нарушая общности, мы считаем, что $\sigma_{\mathrm{d}}\left(K_{1}\right)$ конечен и $\sigma_{\mathrm{d}}\left(K_{2}\right)$ бесконечен. Тогда существует последовательность $\beta_{n}, n \in \mathbb{N}$, собственных значений оператора $K_{2}$ такая, что $\lim _{n \rightarrow \infty} \beta_{n}=0$.

Пусть $\alpha \in \sigma_{\mathrm{d}}\left(K_{1}\right)$. Тогда $\alpha+\beta_{n} \in \sigma(T), n \in \mathbb{N}$. Будем выбрасывать из последовательности $\omega_{n}=\alpha+\beta_{n}, n \in \mathbb{N}$, те члены, для которых $\omega_{\kappa} \in \sigma_{\mathrm{d}}\left(K_{1}\right)$. Тогда количество выброшенных членов $\omega_{1}^{\prime}, \omega_{2}^{\prime}, \ldots$ из $\left\{\omega_{n}\right\}_{n \in \mathbb{N}}$ конечно, так как само множество $\sigma_{\mathrm{d}}\left(K_{1}\right)$ конечно. Обозначим через $\left\{\omega_{\kappa}^{(1)}\right\}_{\kappa \in \mathbb{N}}$ подпоследовательность из $\left\{\omega_{n}\right\}_{n \in \mathbb{N}}$ таких, что $\omega_{\kappa}^{(1)} \notin \sigma_{\mathrm{d}}\left(K_{1}\right)$ для всех $\kappa \in \mathbb{N}$. Теперь будем выбрасывать из $\left\{\omega_{\kappa}^{(1)}\right\}_{\kappa \in \mathbb{N}}$ те члены, для которых $\omega_{\kappa}^{(1)} \in \sigma_{\mathrm{d}}\left(K_{2}\right)$. Однако количество выброшенных членов $\omega_{1}^{\prime \prime}, \omega_{2}^{\prime \prime}, \ldots$ 
из $\left\{\omega_{\kappa}^{(1)}\right\}_{\kappa \in \mathbb{N}}$ будет конечно, иначе существовала бы последовательность $\left\{\omega_{\kappa}^{\prime \prime}\right\}_{\kappa \in \mathbb{N}}$ собственных значений компактного оператора $K_{2}$ такая, что $\lim _{\kappa \rightarrow \infty} \omega_{\kappa}^{\prime \prime}=\alpha \neq 0$, а это противоречит компактности оператора $K_{2}$.

Обозначим через $\left\{\omega_{\kappa}^{(2)}\right\}_{\kappa \in \mathbb{N}}$ последовательность, состоящую из членов последовательности $\left\{\omega_{\kappa}^{(1)}\right\}_{\kappa \in \mathbb{N}}$, такую, что $\omega_{\kappa}^{(2)} \notin \sigma_{\mathrm{d}}\left(K_{2}\right)$ для всех $\kappa \in \mathbb{N}$. Следовательно, имеем $\omega_{\kappa}^{(2)}=\alpha+\beta_{n_{\kappa}} \in \sigma_{\mathrm{d}}(T)$ для всех $\kappa \in \mathbb{N}$, где $\beta_{n_{\kappa}}-$ подпоследовательность последовательности $\left\{\beta_{n}\right\}_{n \in \mathbb{N}} \subset \sigma_{\mathrm{d}}\left(K_{2}\right)$. Отсюда $\left|\sigma_{\mathrm{d}}(T)\right|=\infty$, что противоречит сделанному предположению.

ПреДЛОжЕНИЕ 3.3. Дискретный спектр (существенный спектр) оператора T бесконечен тогда и только тогда, когда дискретный спектр оператора $K_{1}$ или $K_{2}$ бесконечен.

\section{4. ЭФФЕКТ ЕФИМОВА}

Существенный спектр $\sigma_{\mathrm{e}}(H)$ оператора $H$ представляется в виде (см. теорему 3.3 из работы [12])

$$
\sigma_{\mathrm{e}}(H)=\sigma\left(H_{0}\right) \cup \sigma\left(H_{0}-T_{1}\right) \cup \sigma\left(H_{0}-T_{2}\right) .
$$

В дальнейшем предположим, что в модели (1) $k_{0}(x, y)$ - произвольная неотрицательная функция на $\Omega_{1} \times \Omega_{2}$ и $k_{0}^{-1}(\{0\}) \cap \Omega_{1} \times \Omega_{2} \neq \varnothing$ и $K_{1} \geqslant 0, K_{2} \geqslant 0$.

Tеорема 4.1. Пусть $H_{0} \geqslant\left(E_{\min }(H)+\eta_{0}\right) E$, где $\eta_{0}=\sup \sigma_{\mathrm{e}}(T)$. Ecли дискретный спектр $\sigma_{\mathrm{d}}(T)$ оператора $T(2)$ конечен, то количество собственных значений в модели (1), лежащих ниже нижнего края $E_{\min }(H)$ существенного спектра $\sigma_{\mathrm{e}}(H)$, конечно.

ДоказАТЕЛьство. Имеем

$$
\sigma_{\mathrm{e}}\left(\eta_{0} E-T\right)=\left\{\omega: \omega=\eta_{0}-\lambda, \lambda \in \sigma_{\mathrm{e}}(T)\right\} .
$$

Тогда $\sigma_{\mathrm{e}}\left(\eta_{0} E-T\right) \subset[0, \infty)$ и $0 \in \sigma_{\mathrm{e}}\left(\eta_{0} E-T\right)$. Отсюда $E_{\min }(T)=E_{\min }\left(\eta_{0} E-T\right)=0$. Получаем, что

$$
E_{\min }\left(H-E_{\min }(H) \cdot E\right)=E_{\min }\left(\eta_{0} E-T\right)=0 .
$$

Из $H_{0} \geqslant\left(E_{\min }(H)+\eta_{0}\right) E$ следует, что $\eta_{0} E-T \leqslant H-E_{\min }(H) \cdot E$. Тогда в силу неравенства (4)

$$
\mu_{\kappa}\left(\eta_{0} E-T\right) \leqslant \mu_{\kappa}\left(H-E_{\min }(H) \cdot E\right), \quad \kappa \in \mathbb{N} .
$$

Пусть $\left|\sigma_{\mathrm{d}}(T)\right|=m$. Тогда для множества

$$
\sigma_{\mathrm{d}}^{0}\left(\eta_{0} E-T\right)=\left\{\omega: \omega \in \sigma_{\mathrm{d}}\left(\eta_{0} E-T\right), \omega<0\right\}
$$

имеем

$$
1 \leqslant\left|\sigma_{\mathrm{d}}^{0}\left(\eta_{0} E-T\right)\right|=m_{0} \leqslant m,
$$

т.е. число собственных значений оператора $\eta_{0} E-T$, лежащих ниже нижнего края существенного спектра $\sigma_{\mathrm{e}}\left(\eta_{0} E-T\right)$, не больше $m$. 
Следовательно, в силу теоремы 2.1 существует натуральное число $n$ такое, что

$$
\mu_{n+\kappa}\left(\eta_{0} E-T\right)=0
$$

для всех $\kappa \in \mathbb{N} \cup\{0\}$. Отсюда имеем $\mu_{n+\kappa}\left(H-E_{\min }(H) \cdot E\right)=0$ для всех $\kappa \in \mathbb{N} \cup\{0\}$, т.е. количество отрицательных собственных значений оператора $H-E_{\min }(H) \cdot E$ конечно. Следовательно, количество собственных значений оператора $H$, лежащих ниже нижнего края $E_{\min }(H)$ существенного спектра, также будет конечным.

Из теоремы 4.1 следует

Теорема 4.2. Пусть $H_{0} \geqslant\left(E_{\min }(H)+\eta_{0}\right) E$. Чтобы в модели (1) ниже нижнего края $E_{\min }(H)$ существенного спектра $\sigma_{\mathrm{e}}(H)$ существовал эфбект Ефимова, необходимо, чтобы оператор $T$ имел бесконечный дискретный спектр, m.е. $\left|\sigma_{\mathrm{d}}(T)\right|=\infty$.

В пространстве $L_{2}\left(\Omega_{1} \times \Omega_{2}\right)$ определим самосопряженные операторы $W_{1}$ и $W_{2}$ :

$$
W_{1}=H_{0}-T_{1}, \quad W_{2}=H_{0}-T_{2} \text {. }
$$

Надо отметить, что дискретный спектр у операторов $W_{1}$ и $W_{2}$ отсутствует. Если $f(x, y)$ - собственная функция оператора $W_{1}$, т.е. $W_{1} f=\lambda f$ для некоторого $\lambda \in \mathbb{R}$, то для функций $g(x, y)=\psi(y) f(x, y)$ имеем $W_{1} g(x, y)=\lambda g(x, y)$, где $\psi(y)$ - произвольная существенно ограниченная измеримая функция, заданная на $\Omega_{2}$. Следовательно, всякое собственное значение оператора $W_{1}$ является бесконечнократным, т.е. $\sigma_{\mathrm{d}}\left(W_{1}\right)=\varnothing$. Аналогично можно показать, что $\sigma_{\mathrm{d}}\left(W_{2}\right)=\varnothing$.

Из теоремы 3.3 работы [12] следует, что для нижнего края $E_{\min }(H)$ существенного спектра оператора $H$ имеется два случая: $E_{\min }(H)=E_{\min }\left(W_{1}\right)$ или $E_{\min }(H)=$ $E_{\min }\left(W_{2}\right)$.

Tеорема 4.3. Пусть $E_{\min }(H)=E_{\min }\left(W_{1}\right)=\Lambda_{1} \leqslant E_{\min }\left(W_{2}\right)$. Если существует ортонормированная система $\left\{\varphi_{n}\right\}_{n \in \mathbb{N}} \subset L_{2}\left(\Omega_{2}\right)$, удовлетворяющая условию

$$
\left(W_{1} f_{\kappa}, f_{\kappa}\right)<\Lambda_{1}+\left(T_{2} f_{\kappa}, f_{\kappa}\right), \quad \kappa \in \mathbb{N},
$$

где $f_{\kappa}(x, y)=\varphi_{0}(x) \varphi_{\kappa}(y), \varphi_{0}(x) \equiv 1$, то ниже нижнего края существенного спектра оператора $H$ существует эфбект Ефимова.

ДокАЗАТЕЛЬство. Пусть в пространстве $L_{2}\left(\Omega_{2}\right)$ существует ортонормированная система $\left\{\varphi_{n}\right\}_{n \in \mathbb{N}}$, удовлетворяющая условию (5). Легко проверить, что

$$
\left\|T_{2} f_{n}\right\|=\sqrt{\nu_{1}\left(\Omega_{1}\right)}\left\|K_{2} \varphi_{n}\right\|, \quad n \in \mathbb{N}
$$

В силу компактности оператора $K_{2}$ имеем $\lim _{n \rightarrow \infty}\left\|K_{2} \varphi_{n}\right\|=0$. Однако

$$
\sigma\left(W_{1}\right)=\sigma_{\mathrm{e}}\left(W_{1}\right)
$$

Отсюда и из неравенства (5) получим, что

$$
\Lambda_{1} \leqslant\left(W_{1} f_{n}, f_{n}\right)<\Lambda_{1}+\left(T_{2} f_{n}, f_{n}\right), \quad n \in \mathbb{N} .
$$


Следовательно, $\lim _{n \rightarrow \infty}\left(W_{1} f_{n}, f_{n}\right)=\Lambda_{1}$. Значит, $\lim _{n \rightarrow \infty}\left(H f_{n}, f_{n}\right)=\Lambda_{1}$. С другой стороны, $\left(H f_{n}, f_{n}\right)<\Lambda_{1}, n \in \mathbb{N}$. Тогда, не нарушая общности, можно предположить, что система $\left\{f_{n}\right\}_{n \in \mathbb{N}}$ "упорядочена" в следующем смысле:

$$
\left(H f_{\kappa}, f_{\kappa}\right) \leqslant\left(H f_{\kappa+1}, f_{\kappa+1}\right), \quad \kappa \in \mathbb{N} .
$$

Имеем

$$
S_{\min }(H)=\mu_{1}(H) \leqslant\left(H f_{1}, f_{1}\right)<\Lambda_{1},
$$

т.е. существует $g_{1} \in L_{2}\left(\Omega_{1} \times \Omega_{2}\right),\left\|g_{1}\right\|=1$, такая, что $\mu_{1}(H)=\left(H g_{1}, g_{1}\right)<\Lambda_{1}$ и по теореме о принципе минимакса число $\mu_{1}(H)$ является собственным значением оператора $H$.

Для каждого $\kappa \in \mathbb{N}$ определим подпространство $L_{\kappa} \subset L_{2}\left(\Omega_{1} \times \Omega_{2}\right)$ :

$$
L_{\kappa}=\left\{f \in L_{2}\left(\Omega_{1} \times \Omega_{2}\right):\left(f, f_{j}\right)=0, j=1,2, \ldots, \kappa\right\} .
$$

Пусть $H_{\kappa}$ - сужение оператора $H$ на подпространство $L_{\kappa}, \kappa \in \mathbb{N}$. Для оператора $H_{1}$ имеем

$$
\mu_{1}\left(H_{1}\right)=S_{\min }\left(H_{1}\right)=\inf \left\{\left(H_{1} f, f\right): f \in L_{1},\|f\|=1\right\} \leqslant\left(H_{1} f_{2}, f_{2}\right)=\left(H f_{2}, f_{2}\right)<\Lambda_{1} .
$$

Так как $f_{2} \in L_{1},\left\|f_{2}\right\|=1$, то существует $g_{2} \in L_{1},\left\|g_{2}\right\|=1$, такая, что $\mu_{1}\left(H_{1}\right)=$ $\left(H_{1} g_{2}, g_{2}\right)=\left(H g_{2}, g_{2}\right)<\Lambda_{1}$.

Для каждого $\kappa \geqslant 2$, повторяя аналогичное рассуждение, получаем

$$
\begin{aligned}
\mu_{1}\left(H_{\kappa}\right) & =S_{\min }\left(H_{\kappa}\right)=\inf \left\{\left(H_{\kappa} f, f\right): f \in L_{\kappa},\|f\|=1\right\} \leqslant \\
& \leqslant\left(H_{\kappa} f_{\kappa+1}, f_{\kappa+1}\right)=\left(H f_{\kappa+1}, f_{\kappa+1}\right)<\Lambda_{1},
\end{aligned}
$$

поскольку $f_{\kappa+1} \in L_{\kappa},\left\|f_{\kappa+1}\right\|=1, \kappa \geqslant 2$. Тогда существует $g_{\kappa+1} \in L_{\kappa},\left\|g_{\kappa+1}\right\|=1$, такая, что

$$
\mu_{1}\left(H_{\kappa}\right)=\left(H_{\kappa} g_{\kappa+1}, g_{\kappa+1}\right)=\left(H g_{\kappa+1}, g_{\kappa+1}\right)<\Lambda_{1}
$$

Таким образом, для каждого $\kappa \in \mathbb{N}$ оператор сужения $H_{\kappa}$ имеет собственные значения

$$
\mu_{1}\left(H_{\kappa}\right)<\Lambda_{1}, \quad \mu_{1}\left(H_{\kappa}\right) \leqslant \mu_{1}\left(H_{\kappa+1}\right), \quad \kappa \in \mathbb{N} .
$$

Следовательно, каждое число $\omega_{\kappa}=\mu_{1}\left(H_{\kappa}\right)<\Lambda_{1}, \kappa=0,1,2, \ldots$, является собственным значением оператора $H$, где $H_{0}=H$ и $\lim _{n \rightarrow \infty} \omega_{n}=\Lambda_{1}$.

Tеорема 4.4. Пусть $E_{\min }(H)=E_{\min }\left(W_{2}\right)=\Lambda_{2} \leqslant E_{\min }\left(W_{1}\right)$. Если существует ортонормированная система $\left\{\varphi_{n}\right\}_{n \in \mathbb{N}} \subset L_{2}\left(\Omega_{1}\right)$, удовлетворяющая условию

$$
\left(W_{2} f_{\kappa}, f_{\kappa}\right)<\Lambda_{2}+\left(T_{1} f_{\kappa}, f_{\kappa}\right), \quad \kappa \in \mathbb{N},
$$

где $f_{\kappa}(x, y)=\varphi_{\kappa}(x) \varphi_{0}(y), \varphi_{0}(y) \equiv 1$, то ниже нижнего края существенного спектра оператора $H$ существует эффект Ефимова. 


\section{5. ПРИМЕР}

Рассмотрим последовательность $p_{0}=0, p_{1}=1 / 2, p_{n}=p_{n-1}+1 / 2^{n}, n \in \mathbb{N}$. Положим

$$
q_{n}=\frac{p_{n}-p_{n-1}}{2}, \quad n \in \mathbb{N} .
$$

На $[0,1]$ определим функцию $u(x)$

$$
u(x)=\left\{\begin{array}{lll}
0, & \text { если } & x \in[0,1 / 2], \\
u_{0}(x), & \text { если } & x \notin[0,1 / 2],
\end{array}\right.
$$

где $u_{0}(x)=\sum_{n \in \mathbb{N}} \delta_{n} r_{n}(x)$,

$$
\begin{gathered}
r_{\kappa}(x)=\left\{\begin{array}{lll}
\frac{p_{\kappa}-x}{p_{\kappa}-q_{\kappa+1}}, & \text { если } & x \in\left[p_{\kappa}, q_{\kappa+1}\right], \\
\frac{p_{\kappa+1}-x}{p_{\kappa+1}-q_{\kappa+1}}, & \text { если } & x \in\left[q_{\kappa+1}, p_{\kappa+1}\right], \\
0, & \text { если } & x \notin\left[p_{\kappa}, p_{\kappa+1}\right],
\end{array}\right. \\
\delta_{1}=1, \quad \delta_{n} \leqslant\left(\frac{\sqrt{2}}{3}\right)^{n}, \quad n \geqslant 2 .
\end{gathered}
$$

В пространстве $L_{2}[0,1]$ рассмотрим последовательность ортонормированных функций

$$
\varphi_{n}(y)=2^{(n+1) / 2} \sin \xi_{n}(y), \quad n \in \mathbb{N},
$$

где

$$
\xi_{\kappa}(y)=\left\{\begin{array}{lll}
\frac{\pi}{p_{\kappa}-p_{\kappa-1}}\left(y-p_{\kappa-1}\right), & \text { если } & y \in\left[p_{\kappa-1}, p_{\kappa}\right], \\
0, & \text { если } & y \notin\left[p_{\kappa-1}, p_{\kappa}\right] .
\end{array}\right.
$$

Определим ядро $k_{2}(y, t)$ :

$$
k_{2}(y, t)=\sum_{n \in \mathbb{N}}\left(\frac{2}{3}\right)^{n} \varphi_{n}(y) \varphi_{n}(t) .
$$

Ряд (7) равномерно сходится в квадрате $[0,1]^{2}$. Следовательно, интегральный оператор $K_{2}$, заданный ядром $k_{2}(y, t)$, самосопряжен и положителен в $L_{2}[0,1]$.

В пространстве $L_{2}[0,1]$ рассмотрим следующую модель:

$$
H=H_{0}-\left(\gamma T_{1}+T_{2}\right), \quad \gamma \geqslant \frac{2}{3},
$$

где

$$
\begin{aligned}
& H_{0} f(x, y)=u(x) u(y) f(x, y), \\
& T_{1} f(x, y)=\int_{0}^{1} f(s, y) d \nu(s), \\
& T_{2} f(x, y)=\int_{0}^{1} k_{2}(y, t) f(x, t) d \nu(t) .
\end{aligned}
$$


Покажем, что в модели (8) ниже нижнего края существенного спектра существует эффект Ефимова. Рассмотрим последовательность ортонормированных функций $f_{n} \in L_{2}[0,1]^{2}, n \in \mathbb{N}:$

$$
f_{n}(x, y)=\varphi_{0}(x) \varphi_{n}(y), \quad n \in \mathbb{N}
$$

где $\varphi_{0}(x) \equiv 1$. Имеем

$$
\left(\left(H_{0}-\gamma T_{1}\right) f_{1}, f_{1}\right)=\left(H_{0} f_{1}, f_{1}\right)-\gamma\left(T_{1} f_{1}, f_{1}\right)=-\gamma\left(T_{1} f_{1}, f_{1}\right)=-\gamma .
$$

Отсюда и из неравенства

$$
\left(\left(H_{0}-\gamma T_{1}\right) f, f\right) \geqslant-\gamma\left(T_{1} f, f\right) \geqslant-\gamma, \quad f \in L_{2}[0,1]^{2}, \quad\|f\|=1,
$$

получаем, что $E_{\min }\left(H_{0}-\gamma T_{1}\right)=-\gamma$. С другой стороны,

$\left(\left(H_{0}-T_{2}\right) f, f\right)=\left(H_{0} f, f\right)-\left(T_{2} f, f\right) \geqslant-\left(T_{2} f, f\right) \geqslant-\frac{2}{3}, \quad f \in L_{2}[0,1]^{2}, \quad\|f\|=1$.

Следовательно, из $\gamma \geqslant 2 / 3$ и в силу теоремы 3.3 из работы [12] получаем, что $E_{\min }\left(H_{0}-\left(\gamma T_{1}+T_{2}\right)\right)=-\gamma$. Положим $b_{n}=(2 / 3)^{n}, n \in \mathbb{N}$. Тогда каждое число $\omega_{n}=\gamma+b_{n}$ является собственным значением оператора $T=\gamma T_{1}+T_{2}$, так как

$$
\begin{aligned}
\left(T f_{n}\right)(x, y) & =\gamma \int_{0}^{1} \varphi_{0}(s) \varphi_{n}(y) d \nu(s)+\int_{0}^{1} k_{2}(y, t) \varphi_{0}(x) \varphi_{n}(t) d \nu(t)= \\
& =\gamma f_{n}(x, y)+b_{n} f_{n}(x, y)=\left(\gamma+b_{n}\right) f_{n}(x, y), \quad n \in \mathbb{N} .
\end{aligned}
$$

Для оператора $H_{0}$ получим, что

$$
\left(H_{0} f_{n}, f_{n}\right)=\int_{0}^{1} u(s) d \nu(s) \int_{0}^{1} u(t) \varphi_{n}^{2}(t) d \nu(t) \leqslant \int_{0}^{1} u(t) \varphi_{n}^{2}(t) d \nu(t) \leqslant \delta_{n}, \quad n \in \mathbb{N} .
$$

Отсюда

$$
\left(\left(H_{0}-\gamma T_{1}\right) f_{n}, f_{n}\right) \leqslant \delta_{n}-\gamma<-\gamma+\left(\frac{2}{3}\right)^{n}=E_{\min }(H)+\left(T_{2} f_{n}, f_{n}\right), \quad n \geqslant 2
$$

т.е. выполняется условие из теоремы 4.3. Значит, в модели (8) ниже нижнего края существенного спектра существует эффект Ефимова.

\section{Список литературы}

[1] В. Н. Ефимов, ЯФ, 12:5 (1970), 1080-1091.

[2] Д. Р. Яфаев, Матем. сб., 94(136):4(8) (1974), 567-593.

[3] Yu. N. Ovchinnikov, I. M. Sigal, Ann. Phys., 123:2 (1979), 274-295.

[4] H. Tamura, J. Funct. Anal., 95:2 (1991), 433-459.

[5] С. Н. Лакаев, ТМФ, 89:1 (1991), 94-104.

[6] С. Н. Лакаев, Функи. анализ и его прил., 27:3 (1993), 15-28.

[7] С.Н. Лакаев, Ж. И. Абдуллаев, Функи. анализ и его прил., 33:2 (1999), 84-88.

[8] Ж. И. Абдуллаев, С. Н. Лакаев, ТМФ, 136:2 (2003), 231-245.

[9] S. Albeverio, S. N. Lakaev, Z.I. Muminov, Ann. Henri Poincaré, 5:4 (2004), 743-772; arXiv: math-ph/0312026. 
[10] С. Н. Лакаев, З.Э. Муминов, Функи. анализ и его прил., 37:3 (2003), 80-84.

[11] A. V. Sobolev, Comm. Math. Phys., 156:1 (1993), 101-126.

[12] Ю. Х. Эшкабилов, ТМФ, 149:2 (2006), 228-243.

[13] Ю.Х. Эшкабилов, О. И. Сахобидинова, "О бесконечности дискретного спектра операторов в модели Фридрихса", Математический форум, т. 1, Исследования по математическому анализу, ВНЦ РАН, Владикавказ, 2008, 246-255.

[14] М. Рид, Б. Саймон, Методъ современной математической физики, т. 1, Функциональный анализ, Мир, М., 1977.

[15] М. Рид, Б. Саймон, Методы современной математической физики, т. 4, Анализ операторов, Мир, М., 1982.

[16] Ю. Х. Эшкабилов, Узбек. матем. журн., 2005, № 3, 104-112.

Поступила в редакцию 29.10.2009 